DOI: 10.25100/eg.v0i23.11713

Espacios y Territorios

\title{
Mapeo de la cicatriz pos-incendio forestal para la estimación de pérdidas potenciales del área de producción de pulpa de papel, Municipios de Yumbo, La Cumbre y Dagua (Valle del Cauca), año 2014
}

\section{Mapping of the post-forest fire scar to estimate potential losses in the paper pulp production area, Municipalities of Yumbo, La Cumbre and Dagua (Valle del Cauca), year 2014}

\author{
Eduard Paz Zúñiga \\ Ingeniero Topográfico, Universidad del Valle. Cali-Colombia. \\ eduardfpaz@gmail.com | 0000-0002-2654-1839
}

\begin{abstract}
Para citar este artículo: Paz Zúñiga. E. (2022). Mapeo de la cicatriz pos-incendio forestal para la estimación de pérdidas potenciales del área de producción de pulpa de papel, Municipios de Yumbo, La Cumbre y Dagua (Valle del Cauca), año 2014. Entorno Geográfico, (23), e20211713. https://doi.org/10.25100/eg.v0i23.11713
\end{abstract}

\section{Resumen}

En este estudio se pretende determinar el área de afectación o cicatriz pos - incendio forestal sobre los cultivos de producción de pulpa de papel en los municipios de Yumbo, La Cumbre y Dagua a partir de imágenes Landsat 8. La metodología parte de escoger imágenes satelitales Landsat 8 con la mínima cobertura de nubes, a las cuales se les realizan las correcciones radiométricas haciendo la conversión de niveles digitales (ND) a valores de radiancia y reflectancia, así como las correcciones atmosféricas y topográficas. Posteriormente se estiman el índice de vegetación normalizado (NDVI), el índice normalizado de diferencia de humedad (NDMI), así como el ajustado al suelo (NMDISoil) y por último el Radio normalizado de incendio (NBR), los cuales se utilizaron para la estimación de la cobertura vegetal antes de la temporada de sequía, la detección de posibles focos de incendio y finalmente para el mapeo de la cicatriz pos incendio, adicionalmente se estimó la temperatura de brillo producto que sirvió para validar los resultados obtenidos con los índices antes mencionados. Al contar con diferentes fechas de captura se obtuvieron varias fases, entre enero y abril se estimó la cobertura vegetal y se determinó por medio de la relación entre el NDVI y la temperatura de brillo las áreas propensas a incendios forestales para la temporada de sequía, en la segunda fase que comprende las capturas entre junio hasta agosto se realizó la detección de incendios forestales por medio de los índices NDMI y NDMISoil, dando como positivo un foco para la imagen capturada el 1 de junio del 2014, adicionalmente para esta misma fecha se mostró el resultado de temperatura calculada, la cual alcanzó temperaturas 
superiores a $50^{\circ}$ Celsius en zonas cercanas al foco detectado, este producto también se obtuvo para las imágenes correspondientes al 20 de agosto y 5 de septiembre, mostrando temperaturas superiores a $30^{\circ}$ Celsius, las cuales corresponde a fechas posteriores a incendios forestales, en la tercera fase que corresponde al mes de septiembre se calculó el NBR, permitiendo el mapeo de la cicatriz pos-incendio, la secuencia de las tres fases termino con la estimación de áreas de producción afectadas, donde se encontró que los cultivos fueron afectados en casi un $38 \%$, los que podría significar pérdida de casi un $50 \%$ en la producción de pulpa.

Palabras clave: incendios forestales, cicatriz pos - incendio, NDVI, NDMI, NDMISoil, NBR y temperatura de brillo.

\section{Abstract}

This study aims to determine the affected area or post-forest fire scar on the paper pulp production crops in the municipalities of Yumbo, La Cumbre and Dagua from Landsat 8 images. The methodology starts from choosing Landsat satellite images 8 with minimal cloud cover, to which radiometric corrections are made by converting digital levels (ND) to radiance and reflectance values, as well as atmospheric and topographic corrections. Subsequently, the normalized vegetation index (NDVI), the normalized moisture difference index (NDMI), as well as the adjusted to the soil (NMDISoil) and finally the normalized fire radius (NBR) are estimated, which were used for the estimation of the vegetation cover before the drought season, the detection of possible sources of fire and finally for the mapping of the post-fire scar, additionally the product brightness temperature was estimated that served to validate the results obtained with the indices before mentioned. By having different capture dates, several phases were obtained, between January and April the vegetation cover was estimated and the areas prone to forest fires for the drought season were determined through the relationship between the NDVI and the brightness temperature, in In the second phase that includes captures between June and August, the detection of forest fires was carried out through the NDMI and NDMISoil indices, giving a positive focus for the image captured on June 1, 2014, additionally for this same date it was shown The calculated temperature result, which reached temperatures higher than $50^{\circ}$ Celsius in areas close to the detected focus, this product was also obtained for the images corresponding to August 20 and September 5, showing temperatures higher than $30^{\circ}$ Celsius, which corresponds to dates after forest fires, in the third phase corresponding to the month of September the NBR was calculated, allowing the $\mathrm{m}$ felling of the post-fire scar, the sequence of the three phases ended with the estimation of affected production areas, where it was found that the crops were affected by almost $38 \%$, which could mean a loss of almost $50 \%$ in the pulp production.

Keywords: forest fires, post-fire scar, NDVI, NDMI, NMDISoil, NBR and brightness temperature.

Recibido: 25 de mayo de 2021

Aceptado: 23 de julio de 2021 


\section{Introducción}

La intervención del hombre en la naturaleza es muy perjudicial y los costos que esta ha tenido que cubrir por varias generaciones la hacen muy susceptible a que se produzcan daños irreversibles, para el caso de cultivos de madera, el riesgo se basa en que un incendio forestal de gran magnitud podría ocasionar pérdidas totales del cultivo y en muchas ocasiones por intervención directa del hombre los desastres naturales son evidentes, teniendo en cuenta la importancia de saber la afectación pos-incendio de un cultivo de madera, en una zona donde se queman anualmente gran cantidad de bosques que pertenecen a este sector, las técnicas de teledetección aplicadas a imágenes satelitales, partiendo de su resolución temporal y espacial que permiten un seguimiento continuo y cubrir grandes extensiones de área son de gran importancia, según Schroeder et al. (2012) Las estimaciones erradas son causadas principalmente por detección de zonas de cosecha es decir expuestas por tala o por afloramientos rocosos que se encuentran sobre la superficie, por lo cual después de implementar los entrenamientos de clasificación basados en los umbrales adaptativos la detección de áreas quemadas es altamente precisa y califica el uso de sensores remotos para esta importante labor en el sector de producción forestal, por lo cual el uso de imágenes satelitales en la generación de cartografía de áreas afectas por incendios forestales se ha convertido en una tendencia debido a su precisión y fácil adquisición. Según Quintano et al. (2011) Los incendios forestales en todo el mundo generan grandes pérdidas en los sectores productivos y una gran descarga de contaminantes al medio ambiente. Establecer las áreas quemadas después de un incendio es de mucha ayuda en la cuantificación de pérdidas económicas y daños al medio ambiente (Krishna, 2012), teniendo en cuenta la magnitud de la afectación, estableciendo la dinámica de propagación y efectuando el monitoreo de los incendios activos, se pudiese determinar cuál es la dirección de afectación (Wang et al. 2008), el correcto monitoreo e interpretación de la dinámica de los incendios forestales son de carácter urgente cuando se presentan estos fenómenos y permite dar respuesta rápida ante una catástrofe de este tipo.

El Valle de Cauca en los últimos años se ha visto altamente afectado por incendios forestales y en lo que va corrido del presente año se tiene una tendencia al alza debido a la sequía que afronta el departamento CVC (2014), según el periódico El Tiempo (2014) en uno de los últimos incendios registrados en el corregimiento de Mulalo el área de afectación fue de casi 1000 ha, teniendo esto presente fue seleccionada esta época como evento de estudio y sectores aledaños de mayor extensión como los Municipios de Yumbo, Caicedonia y Dagua que se han visto gravemente afectados por incendios y tienen gran participación en áreas de cultivo para la generación de pulpa de papel, como zona de evaluación, al ser esta región una de las más significantes para el sector de producción de papel y cartón, generando una producción a nivel nacional del 35.19\% de pulpa de papel del total, se determinara el área afectada durante y después del evento principalmente para los cultivos incidentes en esta producción es decir cultivos de pino y eucalipto perteneciente en esta caso a Smurfit Kappa Cartón de Colombia, debido a la importancia del monitoreo como posterior mapeo de las áreas afectadas en el estudio se tendrán estos dos resultados y posteriormente se estimaran las áreas de producción 
potencial afectadas por el paso del incendio y así se estimaran las pérdidas ocasionadas a este sector.

\section{Materiales y métodos}

\section{Zona de Estudio y Fuentes de información}

El mapeo de áreas quemadas se realizará sobre las zonas de cultivo pertenecientes a Smurfit Kappa Cartón de Colombia, en la zona central del Departamento del Valle de Cauca, delimitado por los flancos de la cordillera occidental. Los Municipios de interés por incendios forestales registradas son Yumbo y Dagua, pero adicionalmente se tendrá el Municipio de La cumbre por estar en medio de estos dos municipios altamente afectados y será tomado también como referencia para estimar su afectación, ver Figura 1.

Se obtuvieron la imágenes satelitales a partir del satélite Landsat 8 del sensor OLI, hasta el momento se tiene definidas como fechas de estudio, en las que ocurrió los últimos incendios forestales sobre los municipios de Yumbo, La Cumbre y Dagua entre los meses de junio, julio y agosto, de los cuales se seleccionaran capturas antes, durante y después de los incendios forestales para generar la cicatriz dejada por el paso de los incendios forestales así como la dinámica de afectación, para lo cual se empezó desde el mes de enero, teniendo en cuenta que se quería evidenciar la afectación de la cicatriz por lo que fue necesario cuantificar las áreas desde el inicio del año, ver Tabla 1.

Fuente: Elaboración Propia a partir de Imagen Satelital/Esri, Maxar, GeoEye, Earthstar Geographics, CNES/Airbus DS, USDA, USGS, AeroGRID, IGN, and the GIS User

\section{Community.}

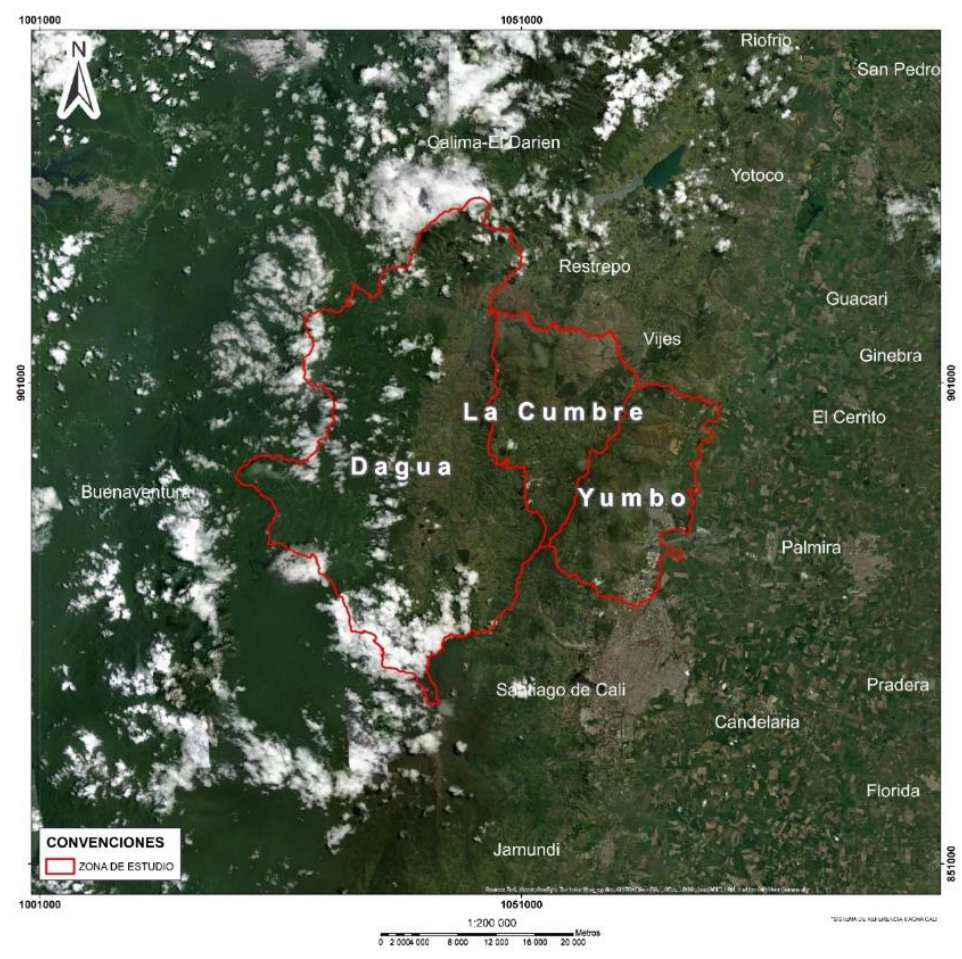

Figura 1: Zona de estudio. 
Tabla 1. Imágenes utilizadas.

\begin{tabular}{|c|c|c|}
\hline Satélite/sensor & Fecha de captura & Uso \\
\hline \multirow{12}{*}{ Landsat 8/OLI } & 2014-01-08 & \multirow{5}{*}{$\begin{array}{c}\text { Reconocimiento de la } \\
\text { cobertura vegetal } \\
\text { (NDVI) }\end{array}$} \\
\hline & 2014-01-24 & \\
\hline & 2014-02-09 & \\
\hline & 2014-03-29 & \\
\hline & 2014-04-14 & \\
\hline & 2014-06-01 & \multirow{5}{*}{$\begin{array}{c}\text { Detección y monitoreo } \\
\text { de incendios y áreas } \\
\text { quemadas }\end{array}$} \\
\hline & 2014-06-17 & \\
\hline & 2014-07-19 & \\
\hline & 2014-08-04 & \\
\hline & 2014-08-20 & \\
\hline & 2014-09-05 & \multirow{2}{*}{$\begin{array}{l}\text { Mapeo de la cicatriz } \\
\text { pos-incendio }\end{array}$} \\
\hline & 2014-09-21 & \\
\hline
\end{tabular}

Fuente: Elaboración propia

Adicionalmente para la determinación de pérdidas en las zonas de producción potencial para Smurfit Kappa Cartón de Colombia se solicitará la cartografía de referencia de los cultivos actuales para el posterior cruce de los resultados de la cartografía existente y así estimar las pérdidas de este sector.

\section{Pre-procesamiento}

Inicialmente se realiza una corrección radiométrica que permite convertir los valores digitales de las imágenes a radiancia mediante la Ecuación 1. Asimismo, para calcular la radiancia de la banda térmica se empleó la Ecuación 2. Posteriormente se estima la reflectancia considerando los modelos empleados por Vanhellemont \& Ruddick (2014) se aplicarán los mismos modelos, Excepto el modelo de conversión a radiancia (Ariza, 2013) y en referencia a los valores de irradiación espectral, se tendrán para cada banda los siguientes, ver Tabla 2.

Tabla 2. Valores de irradiancia espectral para Landsat 8

\begin{tabular}{|l|l|l|}
\hline Banda & \multicolumn{1}{|c|}{ descripción } & Esum o FO \\
\hline 1 & (Coastal/Aerosol) & 1895.6 \\
\hline 2 & (Blue) & 2004.6 \\
\hline 3 & (Green) & 1820.7 \\
\hline 4 & (Red) & 1549.4 \\
\hline 5 & (NIR) & 951.2 \\
\hline 6 & (SWIR1) & 247.6 \\
\hline 7 & (SWIR2) & 85.5 \\
\hline 8 & (PAN) & 1724 \\
\hline 9 & (CIRRUS) & 367 \\
\hline
\end{tabular}

Fuente: Vanhellemont \& Ruddick (2014) 
Teniendo en cuenta los valores anteriores se emplea la siguiente ecuación de Radiancia.

$$
L \lambda=M_{L} * Q_{C A L}+A_{L}
$$

La cual se utilizó para el cálculo de la temperatura de brillo, (ecuación [11]), posterior al uso de esta ecuación se emplean las normalizaciones.

$$
\begin{gathered}
L=\left(\frac{L \max -L \min }{Q \max -Q \min }\right) * N D-Q \min +L \min \\
R=K \pi \frac{L}{E_{\text {sun }} * \cos (\theta)} \\
K=\left[1+0.0167\left(\operatorname{sen}\left(\frac{2 \pi(n-93.5)}{365}\right)\right)\right]^{2}
\end{gathered}
$$

Dónde $\mathrm{L} \lambda=$ Es el valor de Radiancia espectral en el techo de la atmosfera (TOA) medida en valores de (Watts $/ \mathrm{m} 2 * \operatorname{srad} * \mu \mathrm{m})$ ), $\mathrm{ML}=$ Banda - Esun el factor multiplicativo de escalado especifico obtenido del metadato (RADIANCE_MULT_BAND_x, donde x es el número de la banda), $\mathrm{AL}=$ Banda - Esun el factor aditivo de escalado especifico obtenido del metadato (RADIANCE_ADD_BAND_x, donde x es el número de la banda), Lmax y $L \min$ son los valores de Radiancia máximos y mínimos de cada banda, $Q \max$ y $Q \min$ son los valores digitales máximos y mínimos de cada banda, $\mathrm{E}_{\text {sun }}$ corresponde a los valores de irradiación espectral para cada banda, $\theta$ es el ángulo de elevación y $n$ es el día juliano.

La ecuación [1] que permite calcular L $\lambda$ se emplea para convertir los valores o niveles digitales de las imágenes en radiancia. Es decir que se emplea como corrección radiométrica, a su vez, la Ecuación [2] permite calcular la radiancia de la banda térmica para así calcular la temperatura de brillo, utilizando para el cálculo de los índices las ecuaciones [2] y [3], finalmente las combinaciones de los modelos de Landsat 7 y Landsat 8, permiten el cálculo de la temperatura superficial, ecuación [11]

Teniendo en cuenta que la zona de estudio del proyecto está situada sobre terreno montañoso, realizo adicionalmente la Corrección topográfica de las imágenes, para lo cual se utilizara el paquete de Interpreter de Erdas IMAGINE 9.2, en el módulo de Topographic Analysis en la opción de Topographic Normalize, donde se normalizara la zona de estudio, para este procedimiento se utilizara como MDE el suministrado por la NASA, que corresponde a una resolución de pixel de 30m.

\section{Procesamiento}

Para la detección de áreas quemadas según Quintano et al. (2011) Se empieza por el suavizado de la imagen posteriormente se segmenta luego se clasifica y finalmente se mapea, para este caso se utilizarán inicialmente los diferentes índices de entrada definidos por los autores:

Índice normalizado de vegetación NDVI 
Radio normalizado de incendio NBR

Una vez convertidos los valores digitales en el pre-procesamiento, se procede a calcular el NDVI.

$$
N D V I=\frac{(\rho \mathrm{NIR}-\rho \mathrm{RED})}{(\rho \mathrm{NIR}+\rho \mathrm{RED})}
$$

Donde $\rho$ RED es la reflectancia de la banda 4 y $\rho$ NIR la reflectancia de la banda 5 en el sensor OLI de Landsat 8, el cual según sus valores obtenidos tiende a comportase así, valores $>0.72$ muestran una cobertura vegetal sana y gran espesor, mientras valores inferiores o alrededor de 0.14 , puede relacionarse a coberturas bajas y hasta con suelos desnudos, en la siguiente figura se relaciona que, cuando la vegetación es sana la reflectancia en el espectro visible es bajo. Caso contrario cuando los valores son altos en el espectro no visible, ver Figura 2.

Fuente: Earth Observatory (NASA, 2010).

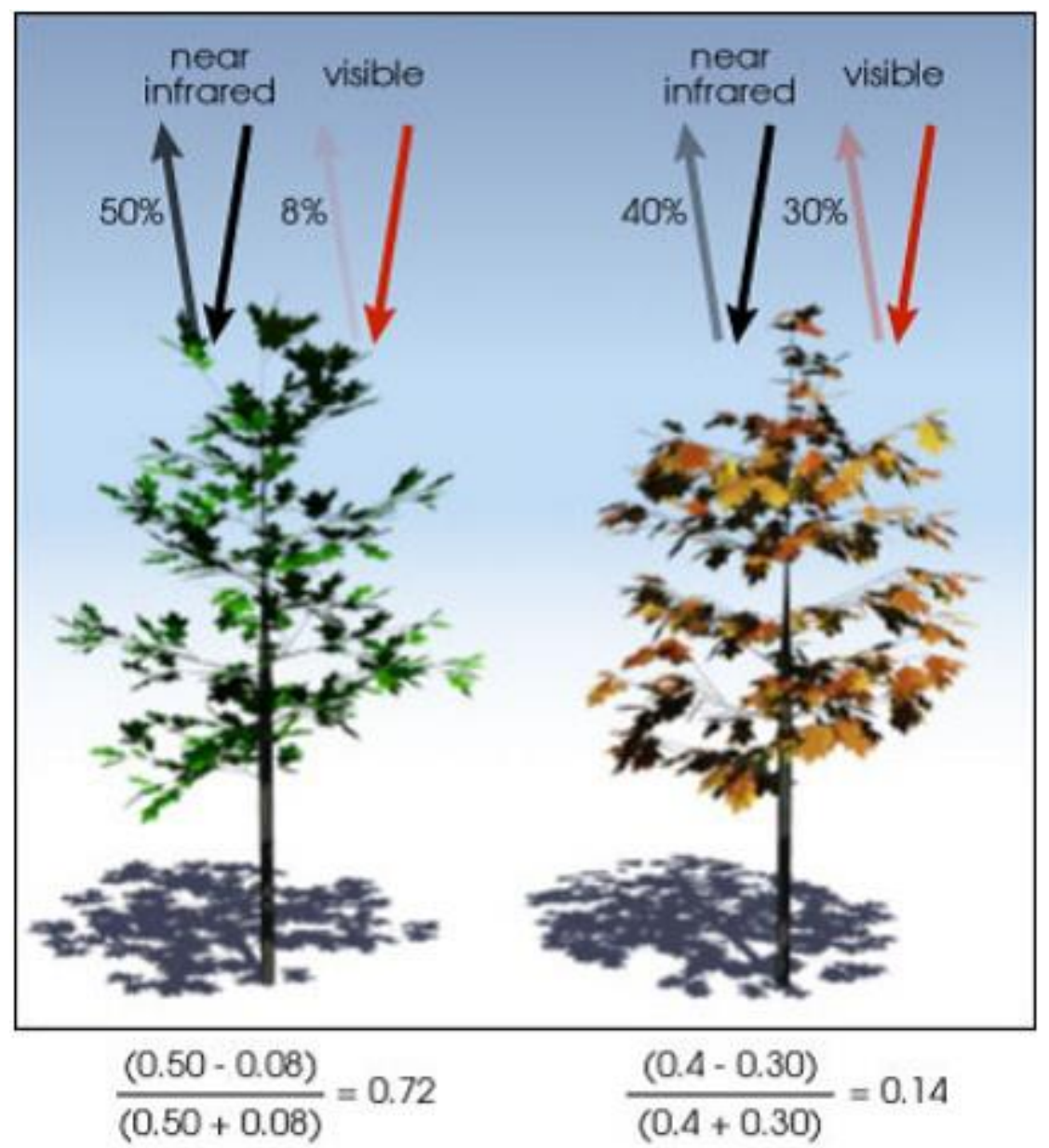

Figura 1. Relación del NDVI con el tipo y estado de vegetación.

Debido a que el área de estudio es una zona altamente afectada por las condiciones atmosféricas según Quintano et al. (2006) la mejor opción es la implementación del algoritmo de INIA-UNAL, para la estimación de las áreas quemadas se utilizó el índice NBR el cual: 


$$
N B R=\frac{(\rho \mathrm{NIR}-\rho \mathrm{SWIR})}{(\sigma \mathrm{NIR}+\rho \mathrm{SWIR})}
$$

Donde $\rho$ SWIR es la reflectancia de la banda 7 y $\rho$ NIR la reflectancia de la banda 5 . Una vez estimado el índice se genera la discriminación de las áreas afectadas por los incendios activos y las áreas finalmente quemadas. Este es un modelo genérico para las imágenes MODIS, sin embargo, se adaptó para Landsat 8 teniendo de referencia los valores espectrales utilizados.

Para el monitoreo de los incendios forestales activos según Wang et al. (2008), se opta por la combinación de las bandas NIR y SWIR, en la obtención del índice NDMI, donde se tiene, que para suelos desnudos los valores más altos de NDMI indican una creciente sequia del suelo, en el caso de áreas de vegetación densa, los valores altos representan una sequía en la vegetación. Mientras que el canal NDMI se comporta así, sucede lo contrario en el canal NDMIsoil, los cuales se calcularon con los siguientes modelos, partiendo de los originales por los autores se adaptaron para Landsat 8/OLI, teniendo en cuenta los valores espectrales de referencia para las bandas seleccionadas.

$$
\begin{gathered}
\mathrm{NDMI}=\frac{R_{(N I R)}-\left(R_{S W I R 6}-R_{S W I R 7}\right)}{R_{(N I R)}+\left(R_{S W I R 6}-R_{S W I R 7}\right)} \\
\text { NDMIsoil }=0.9-\frac{R_{(N I R)}-\left(R_{S W I R 6}-R_{S W I R 7}\right)}{R_{(N I R)}+\left(R_{S W I R 6}-R_{S W I R 7}\right)}[8]
\end{gathered}
$$

Teniendo en cuenta que para algunas de las fechas de estudio se podrán presentar incendios activos la detección de estos se hará según Goodwin y Collett (2014) mediante la implementación del modelo de temperatura de brillo $\left(\mathrm{T}_{\mathrm{L}}\right)$ y temperatura superficial $\left(\mathrm{T}_{\mathrm{S}}\right)$ que utiliza el índice de NDVI, la fracción de bosque $(f v)$ y la emisividad $(\varepsilon)$.

$$
\begin{gathered}
f v=1-\left[\frac{N D V I_{M A X}-N D V I}{N D V I_{M A X}+N D V I_{M I N}}\right]^{a} \\
\varepsilon=f v * \varepsilon_{v}+[1-f v] * \varepsilon_{S} \\
T_{L}=\frac{K_{2}}{\ln \left[\left(\frac{K_{1}}{L_{\lambda}}\right)+1\right]}-273.5 \text { en }^{\circ} \mathrm{C} \\
T_{S}=\left[\frac{T_{L}}{1+\left(\frac{\lambda * T_{L}}{P}\right)} \ln \varepsilon\right] \text { en }{ }^{\circ} \mathrm{C}
\end{gathered}
$$

Donde K1 y K2 Es la constante de conversión K específica para cada banda, dicha constante térmica se suministra en el metadato en este caso al trabajar con Landsat 8 se trabajara con la banda $11, \mathrm{~L}_{\lambda}$ es la Radiancia, $\lambda=11.5^{*} 10-6, \varepsilon_{\mathrm{v}}=0.985$ que es la emisividad de la superficial de la vegetación, $\varepsilon_{s}=0.978$ que es la emisividad superficial del suelo, NDVI $_{M A X}=$ Valor máximo del NDVI, NDVI ${ }_{\text {MIN }}=$ Valor mínimo del NDVI, a=0.6 que se refiere al Valor de la orientación de la hoja planta y $p=\frac{6.626 \times 10^{-34} * 2.998 \times 10^{-8}}{1.38 \times 10^{-23}}$, Donde las constantes corresponde a $6.626 \times 10^{-34}$ Constante de Planck, $2.998 \times 10^{8}$ Velocidad de la luz y $1,38 \times 10^{-23}$ Constante de Boltzman. 


\section{Clasificación}

En la clasificación se agrupan las regiones que muestren el comportamiento de presencia fuego o semilla, el estrechamiento de umbral y el entrenamiento del mismo, permitieron una clasificación más acertada.

Para el caso se opta por una clasificación supervisada teniendo en cuenta que esta entrega los mejores resultados de clasificación, ver Figura 3.

Fuente: Elaboración Propia

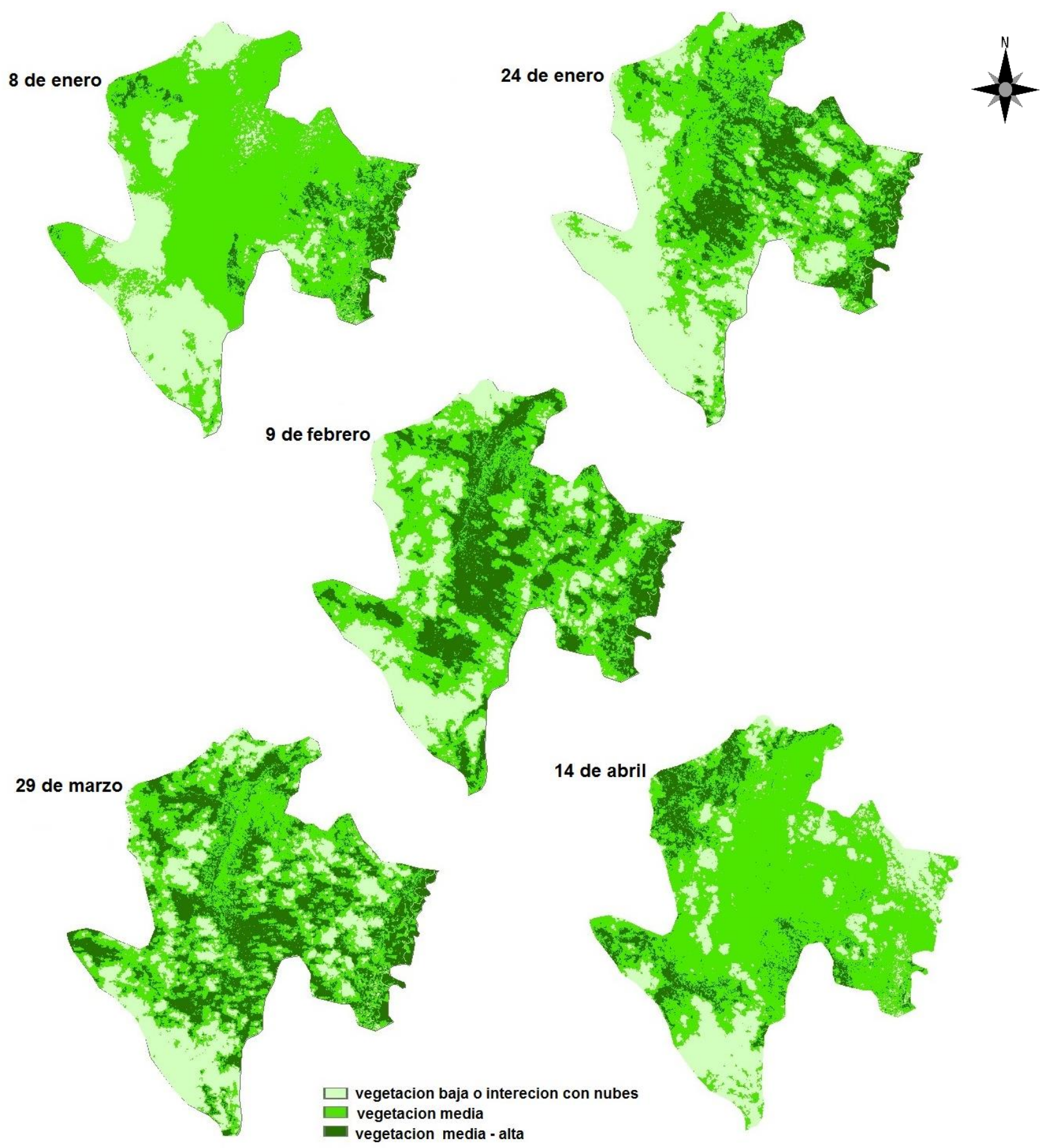

Figura 2. Resultados de NDVI. 


\section{Resultados}

Una vez procesadas las imágenes y aplicados los modelos se obtuvieron los resultados que se utilizaron para completar cada una de las siguientes fases hasta lograr el mapeo de la cicatriz pos-incendio

\section{Estimación de la cobertura vegetal antes-incendio forestal}

Teniendo en cuenta el propósito en cuantificar el área afectada pos-incendio fue necesario realizar una estimación de la cobertura vegetal antes de la incidencia o temporada de sequía, para tal caso, se procesaron las imágenes satelitales capturadas entre los meses de enero hasta abril, ver tabla 1, para las que inicialmente se tuvo en cuenta solo los valores de NDVI (ecuación [5]) obtenidos, ver Figura 2, clasificándolos entre vegetación baja, vegetación media, vegetación medio alta. Teniendo en cuenta que no se tuvieron valores superiores a 0.7 .

Para la recopilación de los resultados antes obtenidos se realizó el compilado de valores de NDVI obteniendo en promedio, los valores acumulados hasta el mes de abril, ver Tabla 3, ver Figura 4. En la cual se realizó una clasificación en tres rangos a partir de los valores obtenidos clasificándolos así.

Tabla 3. Valores de NDVI promedio.

\begin{tabular}{|c|c|c|}
\hline \multicolumn{2}{|c|}{ Valores } & Convención \\
\hline$\square$ & $0-0.007$ & $\begin{array}{c}\text { Interacción con nubes } \\
\text { o vegetación muy baja }\end{array}$ \\
\hline$\square$ & $\begin{array}{c}0.007- \\
0.273\end{array}$ & Vegetación baja \\
\hline & $\begin{array}{c}0.273- \\
0.517\end{array}$ & $\begin{array}{c}\text { Vegetación media - } \\
\text { alta }\end{array}$ \\
\hline
\end{tabular}

Fuente: Elaboración Propia.

Fuente: Elaboración Propia.

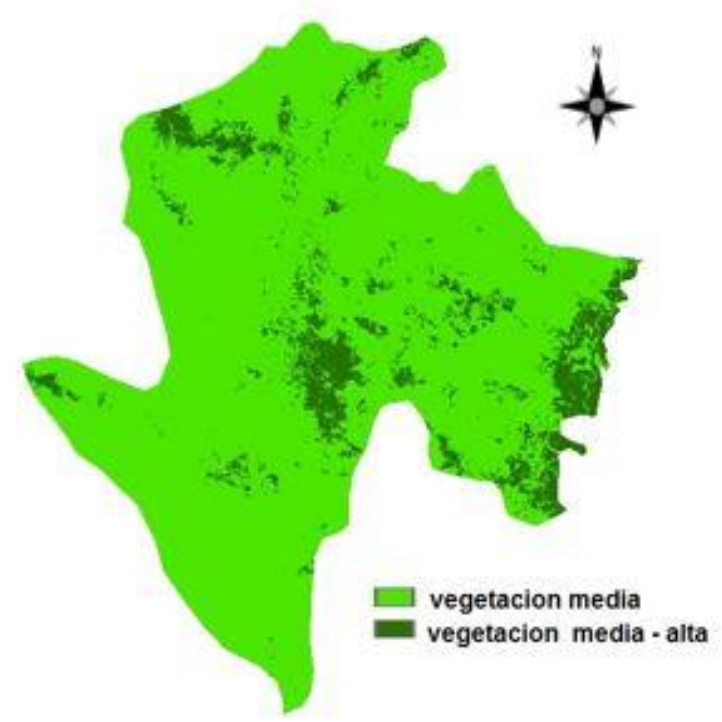

Figura 3. Valores promedio de NDVI. 
Luego de obtener los valores de NDVI, se relacionaron estos con los de temperatura de brillo, los que mostraron cuales serían las áreas que en época seca estarían propensas a incendios forestales, ver Figura 5. Esta apreciación solo se tomó en cuenta para la imagen del 14 de abril del 2014, ya que era la última que se tenía un buen registro antes de empezar en el valle con los incendios masivos.

Fuente: Elaboración Propia.

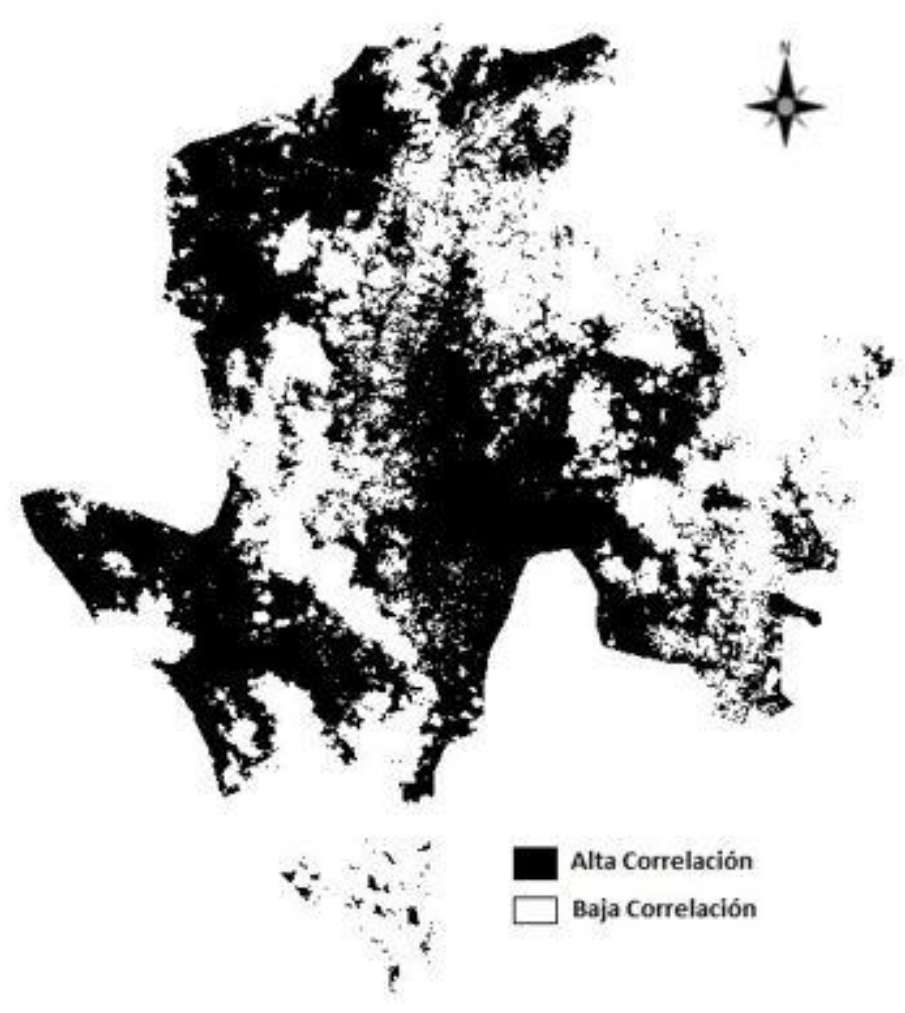

Figura 4. Relación entre NDVI y temperatura de brillo, reconocimiento de zonas propensas a incendios forestales.

\section{Detección de áreas en afectación por incendios}

En esta fase se procesaron las imágenes correspondientes a la actividad masiva de incendios que se registraron, entre los meses de junio y a principios de septiembre del presente año, para realizar la detección de incendios activos y áreas potenciales para que se presente un incendio o se dirija si está ocurriendo a estas zonas, se optó por aplicar el índice de NDMI y NDMISoil (ecuación [7] y [8]), teniendo en cuenta los índices y que son uno el contrario del otro, se mostró el promedio de estos dos en solo tres clases (Ver Figura 6).

Al tener 2 posibles fechas de incendios activos 20 de agosto y el 5 de septiembre se decidieron aplicar a estas imágenes, el cálculo de la temperatura de brillo y temperatura superficial (ecuación [11] y [12]), con el fin de determinar la actividad de incendios forestales (ver Figura 7). 
Fuente: Elaboración Propia.

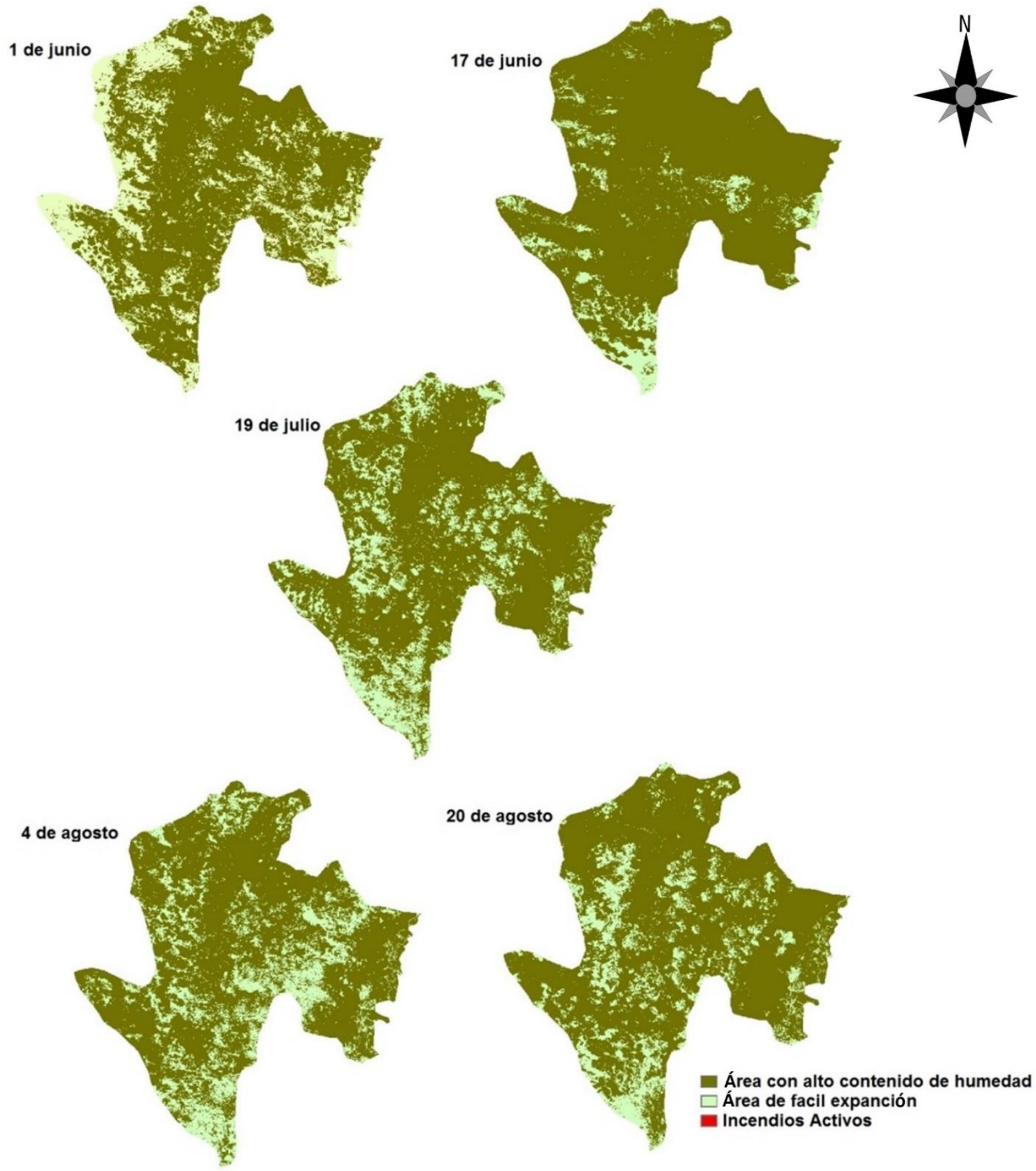

Figura 5. Promedio de Índices de NDMI y NDMISoil.

Los diferentes índices aplicados se obtuvieron por medio de la conversión de ND a valores de radiancia y reflectancia. 


\section{Fuente: Elaboración Propia.}

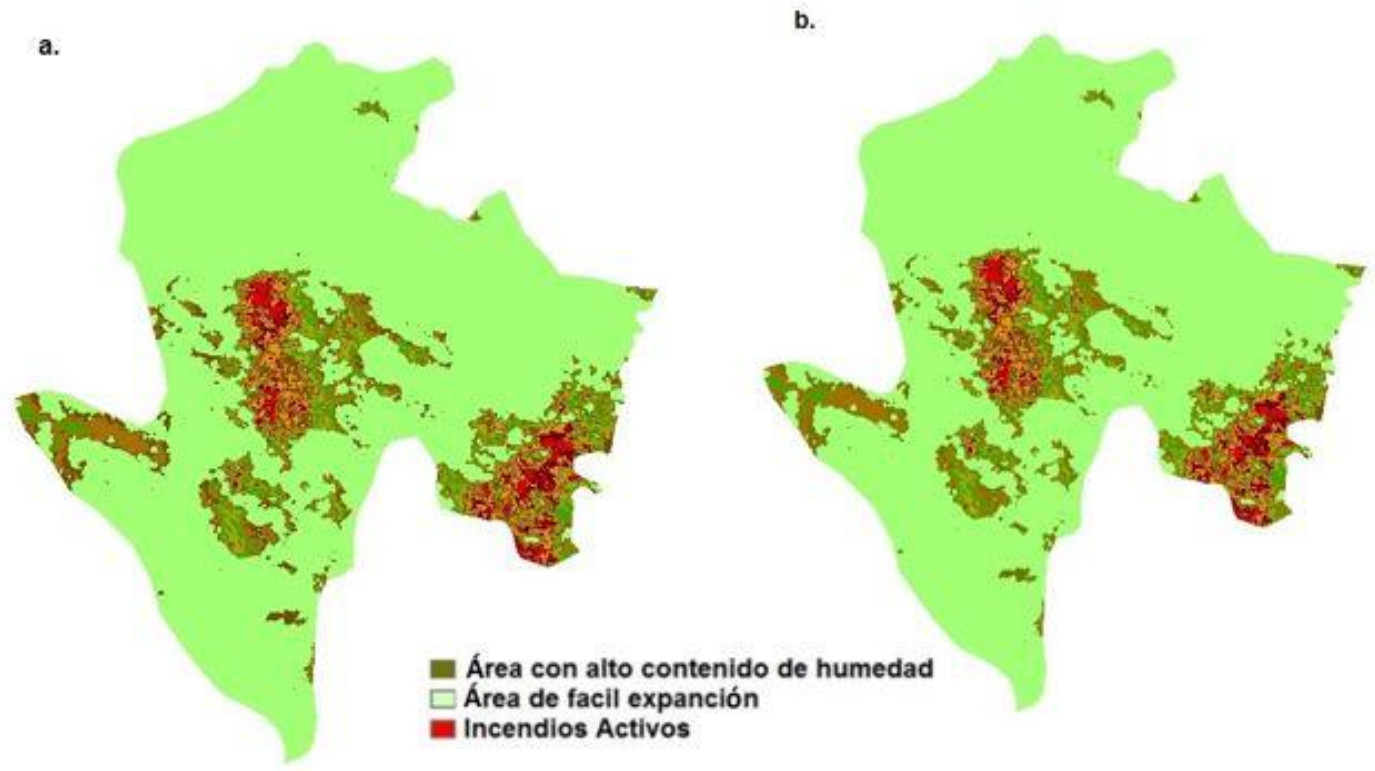

Figura 6. Detección de áreas afectadas por incendios forestales, a. imagen correspondiente al 20 de agosto, b. imagen correspondiente al 5 de septiembre, los colores rojos corresponden a temperaturas superiores a 30 grados. Y muestran la actividad o paso del incendio.

\section{Mapeo de cicatriz pos-incendio}

Una vez obtenidos los anteriores modelos de estimación y detección de incendios se procedió a aplicar el modelo de índice de radio quemado NBR (Ecuación [6]), el cual se aplicó para las imágenes correspondientes al 21 de septiembre que se supone resumirían la cicatriz dejada por los incendios antes registrados, ver Figura 8.

Fuente: Elaboración Propia.

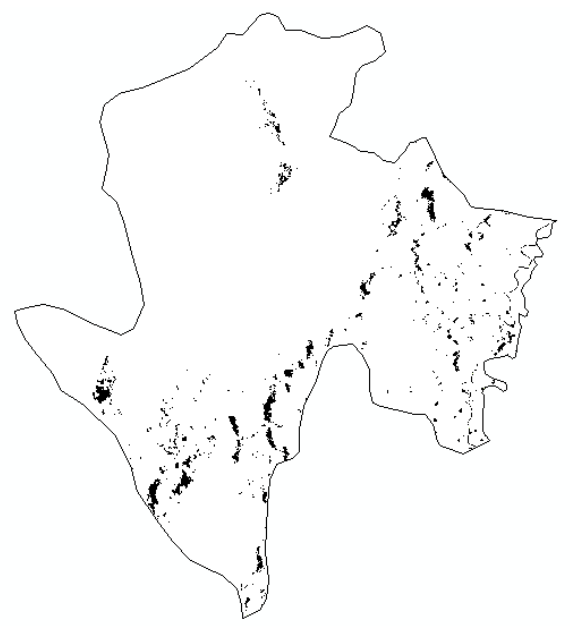

Figura 7. En color negro, Cicatriz dejada por el paso de incendios. 


\section{Discusión}

Después de obtener los diferentes resultados, cabe resaltar que solo se aplicaron los modelos a las imágenes que tenían una referencia específica, por lo cual se lograron los resultados expuestos, que no son los mismos para cada fecha de captura, teniendo en cuenta que el fin era poder llegar hasta el mapeo de la cicatriz pos-incendio, pero no se podía dejar de lado todo lo que antepuso la formación de esta cicatriz, así como el NDVI, que se calculó para llegar a la temperatura de brillo, pero también se utilizó para estimar la cobertura vegetal hasta la temporada de sequía.

Los resultados se utilizaron para varios fines en cada fase del proyecto, inicialmente, se mostró el comportamiento de la cobertura vegetal, en donde se encontraron que muy probablemente por la interacción con la atmosfera los valores de NDVI estaban muy bajos, es decir según la bibliografía consultada solo se alcanzaba una cobertura de tipo media - alta, sin ser esta la característica predomínate en la zona estudiada, lo que llevo a realizar las correcciones atmosféricas en las imágenes procesadas.

Al relacionar el NDVI con la temperatura de brillo para 14 de abril del 2014 se estimaron las zonas propensa a incendios forestales para la temporada de sequía, ver Figura 9, característica que se ratifica inicialmente en la detección de un foco para el 1 de junio del mismo año y también, hace referencias a los eventos que pudieron ser registrados para el 20 de agosto y el 5 de septiembre, fechas que nos corresponde directamente a un evento pero si fueron tomadas después de estos mostrando en el caso de temperatura valores muy elevados.

Fuente: Elaboración Propia.
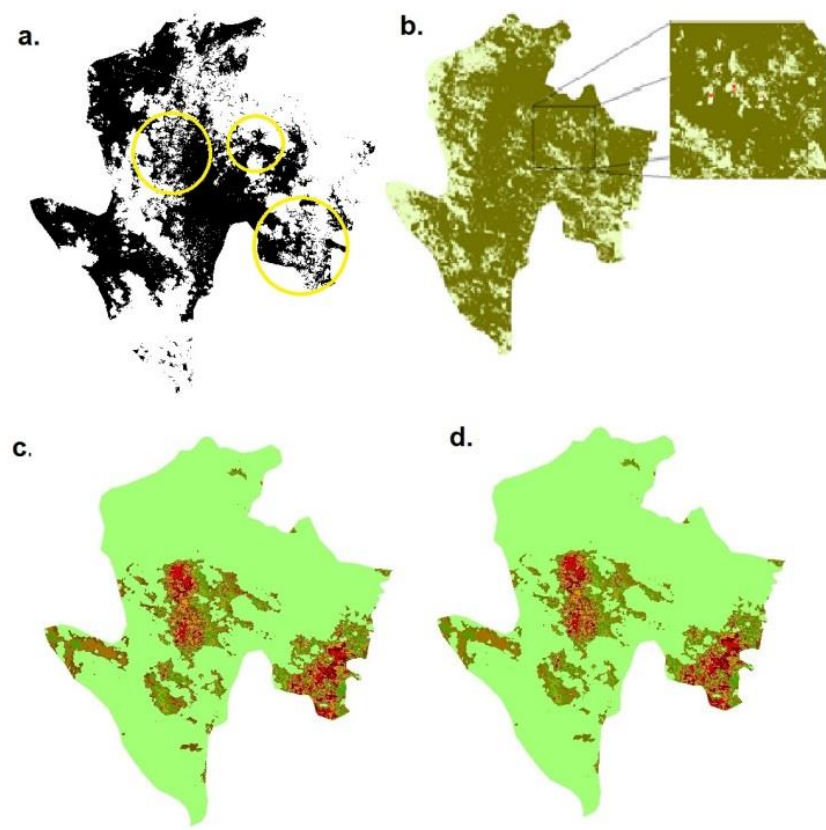

Figura 8. Validación de las áreas propensas a incendios forestales a. Relación entre el NDVI/Temperatura de Brillo, con los eventos posteriormente registrados, b. detección de un foco par el 1 de junio, c. y d. imágenes de temperatura correspondientes al 20 de agosto y 5 de septiembre. 
En el muestreo hecho en la figura anterior con círculos amarillos, se pude ver como posteriormente una de las áreas corresponde a un posible foco y que mucho más adelante, incluso después de casi 4 meses en estas áreas se presentaron incendios, de igual manera se reconoce que el área estimada no es justamente la afectada, pero cabe recordar que esta relación hacer referencia a áreas propensas, que tal como se ve en los resultados obtenidos pueden o no ser afectadas.

Posteriormente en la fase de detección por medio de los índices NDMI y NDMISoil, se detectó un posible foco de incendio forestal, ver Figura 10, característica que no se obtuvo en ninguna otra fecha de captura incluso ni en la del 20 de agosto, en donde justo hasta el día anterior los bomberos habían logrado apagar un incendio que afectó el área de estudio.

Fuente: Elaboración Propia.

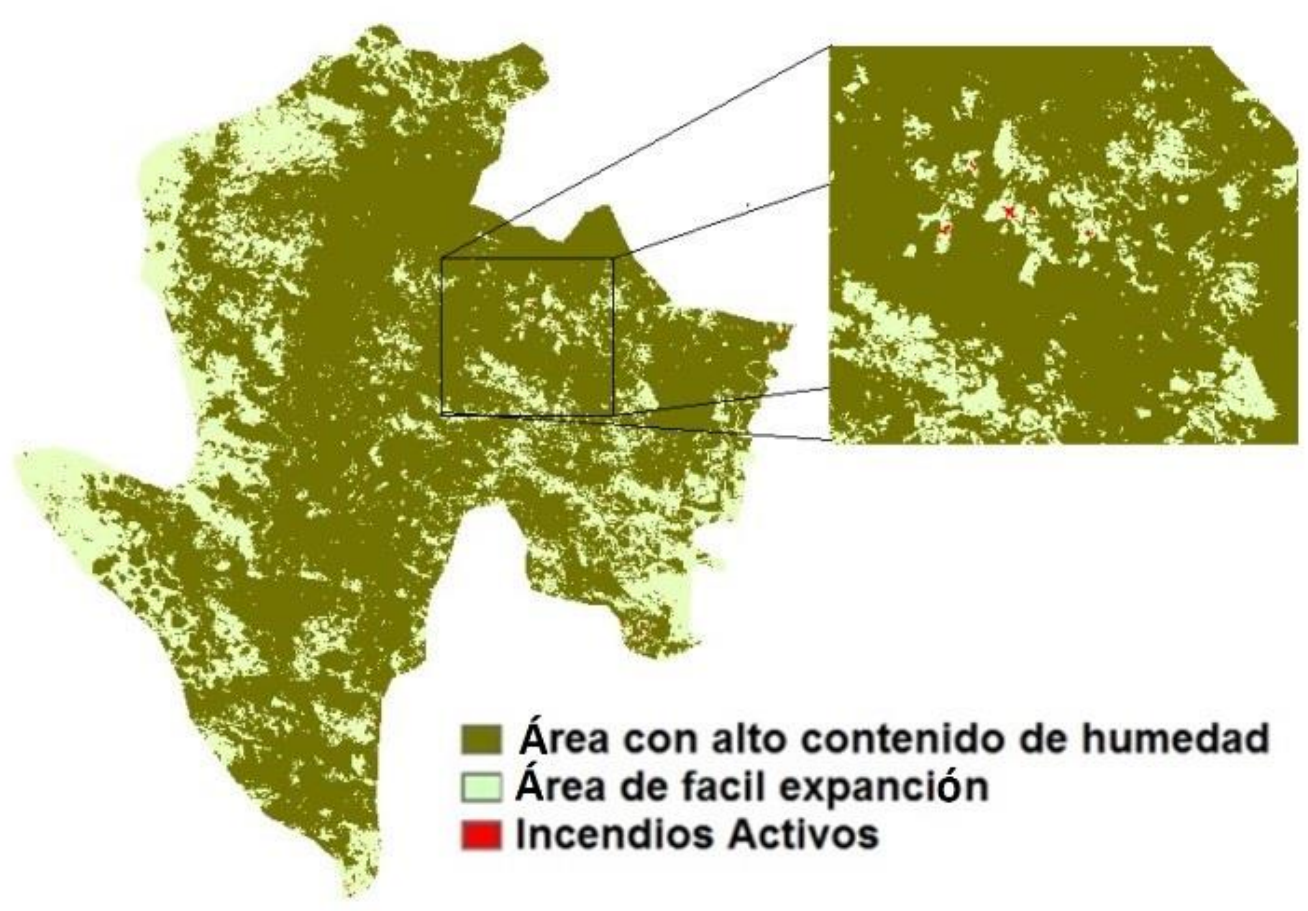

Figura 9. Detección de incendio activo, imagen capturada el 1 de junio del 2014.

$\mathrm{Al}$ encontrar en los resultados o registrar un incendio forestal activo, se decidió aplicar a esta imagen también el modelo de temperatura de brillo, obteniendo lo siguiente, ver Figura 11.

En las diferentes zonas donde se registran temperaturas mayores a $50^{\circ} \mathrm{C}$ y comparar estas zonas con la detectada por índice NDMI, se pude afirmar que toda esa área que está en rojo pudo estar en riesgo eminente de ser alcanzada por el foco del incendio, que tal como se puede ver en las Figuras 9 y 10, el foco detectado esta sobre una de las áreas con temperaturas elevadas.

Al tener el resultado de la cicatriz pos-incendio en color negro en la Figura 8 dejada por el paso del mismo, se encontró que algunas coberturas como el río Cauca habían sido clasificadas como área afectada por el incendio, pero esto muy probablemente se debería a la fuerte respuesta reflectante que tiene el rio. 
Fuente: Elaboración Propia.

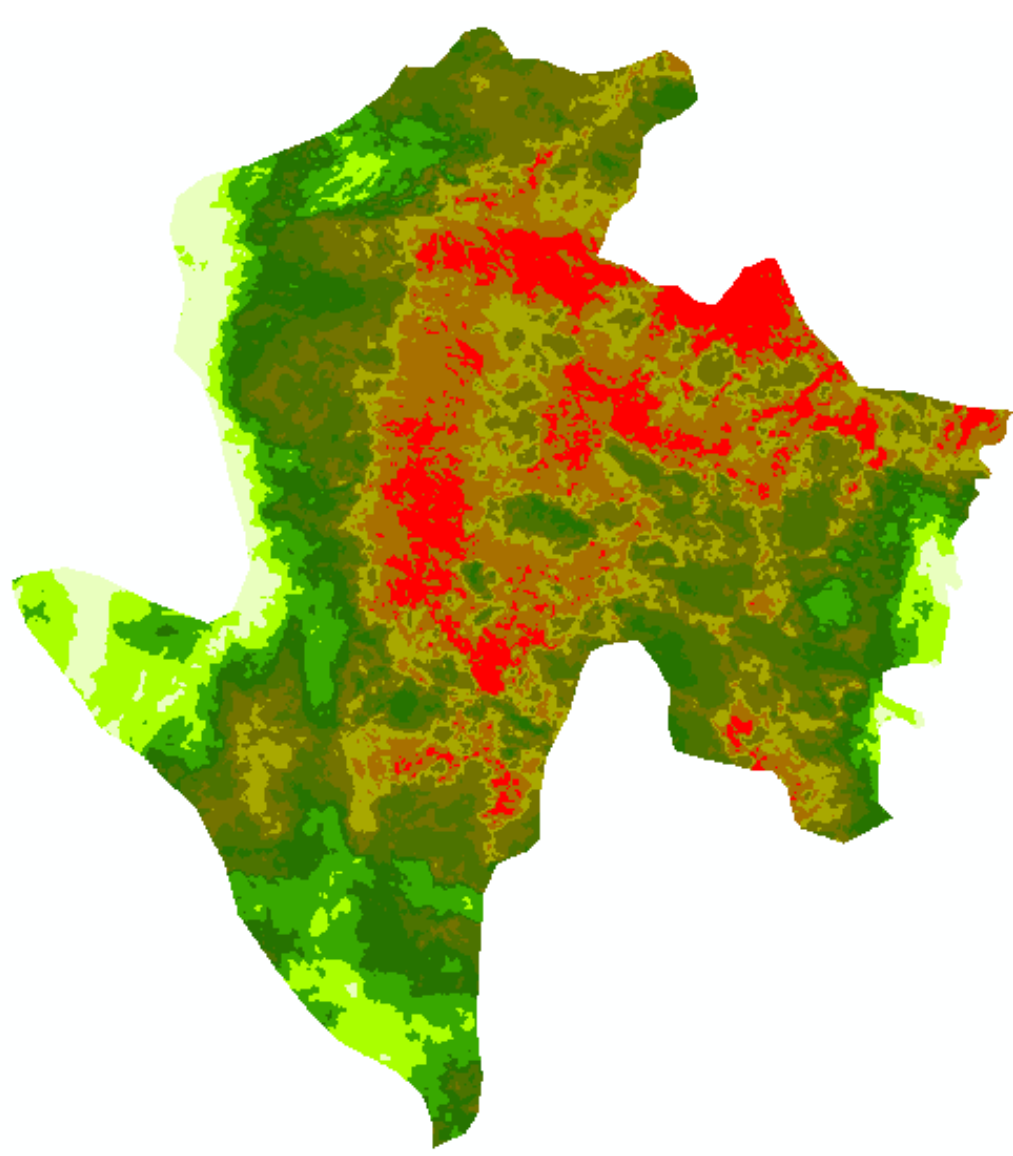

Figura 10. Temperatura de brillo, para el 1 de junio del 2014, en color rojo temperaturas superiores a $50^{\circ}$ Celsius.

Finalmente, al comparar las áreas quemadas con las correspondientes zonas de cultivos para la generación de pulpa de papel, ver Figura 12 se encontró que la afectación, era de casi el $37.8 \%$. Lo cual sería muy significante si no tuviesen cultivos proveedores en otras áreas del país, para la producción interna de pulpa de papel en el valle. 
Fuente: Elaboración Propia.
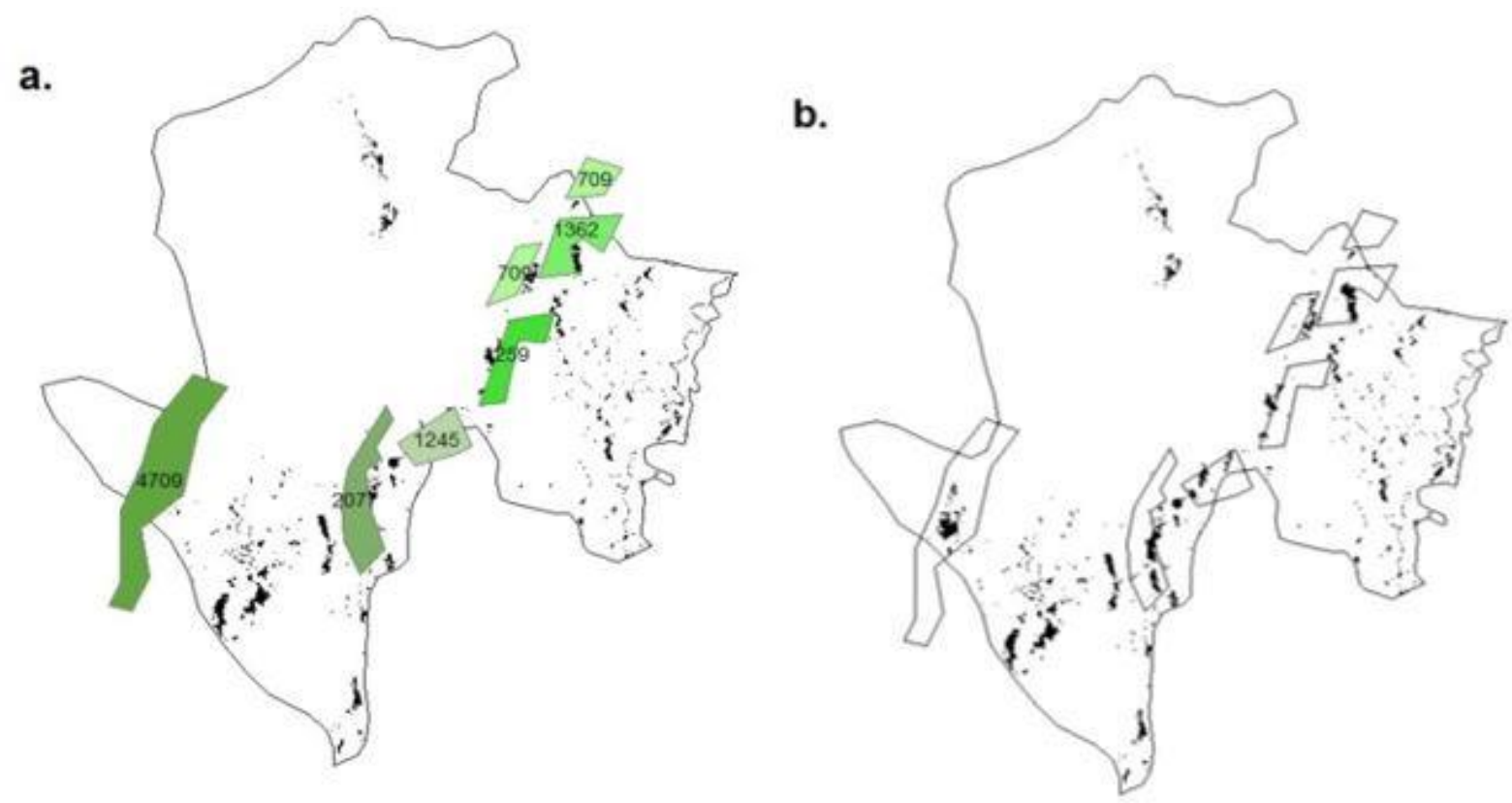

Figura 11. Área de afectación, NBR vs Cultivos de producción de Pulpa, a. cultivos y áreas en hectáreas, b. Zonas de cultivos afectadas.

\section{Conclusiones}

La detección de incendios forestales es tan utilizada en el mundo por medio de técnicas de la teledetección, que incluso con índices tan sencillos como los que se utilizaron en este estudio se entregaron resultados que son de mucha ayuda en el monitoreo, detección, cuantificación y estimación de estos eventos catastróficos.

Al trabajar en una zona con una nubosidad alta, se vieron altamente afectados lo resultados obtenidos, en el caso del NDVI, los valores no superaron 0.6 indicando que no correspondían a vegetación alta y densa, que es lo que más predomina en esta zona exceptuando del área periurbana de Yumbo que en el costado este limita con el valle donde los cultivos predominantes corresponden a la caña de azúcar.

Teniendo en cuenta que se estudiaron diferentes fechas de captura cubriendo en gran parte todo el año 2014, se obtuvieron diferentes resultados, que finalmente se utilizaron en el mapeo de la cicatriz pos-incendio, unos de los productos que se utilizó incluso en la estimación de áreas propensas, detección y monitoreo de incendios, fue la temperatura de brillo. En relación con el NDVI, mostró cuales eran las áreas propensas a incendios forestales, más adelante, presentó gran validez al permitir relacionar el foco encontrado con el índice NDMI para el 1 de junio, con la temperatura, donde se evidenció adicionalmente cual pudo haber sido el área de expansión del incendio activo para ese día de captura.

La temperatura de brillo además de mostrar cómo era la temperatura para la fecha de captura y permitir un monitoreo de la área de expansión, al relacionar el NDVI con este producto se pudo estimar las áreas propensas a incendios, que tal como se mostró más 
adelante para las fechas de captura correspondientes al 1 de junio, 20 de agosto y 5 de septiembre, presentaron un afectación notable, por lo cual se le da gran importancia a este producto ya que su estimación permite obtener varios resultados que son de interés prioritario a la hora de detectar, posteriormente monitorear y muy posiblemente pronosticar acerca de áreas que podrían ser afectadas por incendios.

Al obtener el mapeo de la cicatriz pos-incendio se encontró que el río Cauca estaba siendo clasificado como área quemada, los cual muy posiblemente se debe a su alta reflectividad en donde incluso muchas veces se confunde hasta con carreteras, esto debido a que muestra una baja cobertura vegetal y muy probablemente se tome como suelo desnudo que era uno de los referentes de cicatriz pos-incendio.

Los resultados obtenidos de cicatriz pos incendio mostraron un área de afectación para la industria de la pulpa de papel de casi un $30 \%$ los que indica que la producción puede ser afectada en casi un 50\%, lo cual sería muy perjudicial para el sector de producción de pulpa, pero cabe resaltar que Smurfit Kappa cartón de Colombia no solo tiene cultivos en el Departamento del Valle sino también en el Departamento del Cauca y otros, que hacen posible que el Valle continúe siendo el principal productor de pulpa en el país.

\section{Referencias bibliográficas}

Ariza, A. (2013). Descripcion y correcion de productos Landsat 8. Bogota: Grupo Interno de Trabajo en Percepción Remota y Aplicaciones Geográficas.

Quintano, C., Fernandez, A., Fernández, O., \& Shimabukuro, E. (2006). Mapping burned areas in Mediterranean countries using spectral mixture analysis from a unitemporal perspective. International Journal of Remote Sensing, 27 (4), 645-662. https://doi.org/10.1080/01431160500212195

El Tiempo. (18 de Agosto de 2014). Infierno en las montañas de Yumbo por incendio forestal. $\mathrm{El}$ Tiempo. https://www.eltiempo.com/archivo/documento/CMS14398976

Quintan, C., Fernandéz, A., Stein, A \& Bijker, W. (2011). Estimation of area burned by forest fires in Mediterranean countries: A remote sensing data mining perspective. Forest Ecology and Management, 266 (8), 1597-1607. 10.1016/j.foreco.2011.07.010

CVC. (2014). 56 Incendios forestales se han registrado en el municipio de dagua en los meses de junio y julio. https://bit.ly/3rOsbqb

Krishna, P. H., \& Reddy, C. S. (2012). Assessment of increasing threat of forest fires in Rajasthan, India using multi-temporal remote sensing data (2005-2010). Current Science, 102 (9), 1288-1297.

Lingli Wang, J. J., Qu, J \& Xianjun, H .(2008). Forest fire detection using the normalized multi-band drought index (NMDI) with satellite measurements. ScienceDirect, 148 (11) 1767 - 1776. https://doi.org/10.1016/j.agrformet.2008.06.005

18 
Nicholas R. Goodwin, L. J., \& Collett, L. (2014). Development of an automatedmethod formapping fire history captured in Landsat TM and ETM+ time series across Queensland, Australia. Remote Sensing of Environment, 148 (4) 206-221. 10.1016/j.rse.2014.03.021

Schroeder, T., Wulder, M., Healey, S., \& Moisen, G. (2012). Detecting post-fire salvage logging from Landsat change maps and national fire survey data. Remote Sensing of Environment, 122, 166-174. 10.1016/j.rse.2011.10.031

Vanhellemont, Q., \& Ruddick, K. (2014). Turbid wakes associated with offshore wind turbines observed with Landsat 8. Remote Sensing of Environment, 145, 105-115. https://doi.org/10.1016/j.rse.2014.01.009 